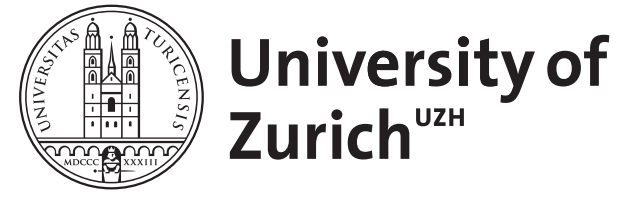

\title{
Population-level consequences of risky dispersal
}

\author{
Shaw, Allison K ; Jalasvuori, Matti ; Kokko, Hanna
}

\begin{abstract}
Achieving sufficient connectivity between populations is essential for persistence, but costs of dispersal may select against individual traits or behaviours that, if present, would improve connectivity. Existing dispersal models tend to ignore the multitude of risks to individuals: while many assess the effect of mortality costs, there is also a risk of failing to find new habitat, especially when the entire inhabitable area remains both small and fragmented. There are few known rules governing whether individuals evolve to disperse more, or less, than what is ideal for population connectivity and persistence. Here we aim to fill this gap, while also noting that evolution might not only produce suboptimal dispersal behaviour: it also influences individual heterogeneity in dispersal. Intuitively, we might expect heterogeneity to improve connectivity, as some individuals will travel far. However, we show that this is only true if dispersal distances on average are quite short; heterogeneity can also lead to reduced connectivity because it can reduce the proportion of the most profitable ('safest') intermediate dispersal distances. In general, our results show that conditions typically associated with conservation concerns (small and fragmented habitats inhabited by a species with a low birth rate) are also ones that are most likely to lead to suboptimal dispersal traits. This prompts the question of assisted dispersal in cases of urgent conservation concern.
\end{abstract}

DOI: https://doi.org/10.1111/oik.01229

Posted at the Zurich Open Repository and Archive, University of Zurich

ZORA URL: https://doi.org/10.5167/uzh-177112

Journal Article

Published Version

Originally published at:

Shaw, Allison K; Jalasvuori, Matti; Kokko, Hanna (2014). Population-level consequences of risky dispersal. Oikos, 123(8):1003-1013.

DOI: https://doi.org/10.1111/oik.01229 
Oikos 123: 1003-1013, 2014

doi: 10.1111/oik.01229

(C) 2014 The Authors. Oikos (C) 2014 Nordic Society Oikos

Subject Editor: Justin Travis. Accepted 22 January 2014

\title{
Population-level consequences of risky dispersal
}

\author{
Allison K. Shaw, Matti Jalasvuori and Hanna Kokko \\ A. K. Shaw (ashaw@umn.edu) and H. Kokko, Div. of Evolution, Ecology and Genetics, Research School of Biology, The Australian National \\ Univ., Canberra ACT 0200, Australia. AKS also at: and Dept of Ecology, Evolution and Behavior, Univ. of Minnesota, St. Paul, MN 55108, \\ USA. - M. Jalasvuori, Centre of Excellence in Biological Interactions, Univ. of Jyväskylä, PO Box 35, FI-40014 University of Jyväskylä, \\ Finland.
}

\begin{abstract}
Achieving sufficient connectivity between populations is essential for persistence, but costs of dispersal may select against individual traits or behaviours that, if present, would improve connectivity. Existing dispersal models tend to ignore the multitude of risks to individuals: while many assess the effect of mortality costs, there is also a risk of failing to find new habitat, especially when the entire inhabitable area remains both small and fragmented. There are few known rules governing whether individuals evolve to disperse more, or less, than what is ideal for population connectivity and persistence. Here we aim to fill this gap, while also noting that evolution might not only produce suboptimal dispersal behaviour: it also influences individual heterogeneity in dispersal. Intuitively, we might expect heterogeneity to improve connectivity, as some individuals will travel far. However, we show that this is only true if dispersal distances on average are quite short; heterogeneity can also lead to reduced connectivity because it can reduce the proportion of the most profitable ('safest') intermediate dispersal distances. In general, our results show that conditions typically associated with conservation concerns (small and fragmented habitats inhabited by a species with a low birth rate) are also ones that are most likely to lead to suboptimal dispersal traits. This prompts the question of assisted dispersal in cases of urgent conservation concern.
\end{abstract}

Habitats of species are rarely entirely homogeneous. Given the amplified risks of extinction of small populations (Soulé 1987), sufficient connectivity between suitable habitats is a requirement for long-term persistence (Hanski 1999). Connectivity is not a property of the landscape alone: it is an emergent property that joins landscape features with a species' dispersal traits (Burgess et al. 2012, Baguette et al. 2013). However, dispersal intertwines individuals and populations together in an eco-evolutionary feedback loop: individuals disperse, influencing the spatial, genetic, social and demographic structure of populations, which feeds back to influence the dispersal strategies favoured by selection (Starrfelt and Kokko 2012).

Given that dispersal is vital for the persistence of any population, the theoretical prediction that evolved dispersal rules often fail to promote population persistence (Delgado et al. 2011) may appear surprising. Dispersal can be favoured by several processes including competition for local resources (particularly competition between related individuals), deteriorating habitat, temporally varying conditions and inbreeding (reviewed by Johnson and Gaines 1990, Ronce 2007, Clobert et al. 2012). Simultaneously, it often comes with substantial costs to the individual: risky movement through unknown habitats, heavy energetic investment, and foregoing familiar resources with no guarantee that new ones will be found (reviewed by Bonte et al. 2012). There is no guarantee that costs and benefits balance in such a manner that would make populations find and utilize available resources in the best possible way ('best' in terms of maximizing connectivity or probability of persistence of a population). In extreme cases, dispersal evolution can even lead to population decline or collapse (Savill and Hogeweg 1998, Gyllenberg et al. 2002, Poethke et al. 2011).

These predictions can be seen as one instance of the well known principle that natural selection does not act on species to improve their persistence, but on lower units of selection. In the case of dispersal, the direction of the mismatch is less obvious than the fact that a mismatch can exist. Perhaps surprisingly, past theory indicates that the population cost can occur in two opposite directions: 1) "inertia", where individuals disperse less than would be best for population persistence, or 2) "hypermobility", where individuals disperse more than would be best for population persistence (Delgado et al. 2011). While inertia and hypermobility have each been demonstrated in a number of past theoretical studies (Table 1), there has not been much effort to document the general conditions under which each is expected to occur. Most of these studies find only either hypermobility or inertia (Roff 1975, Hamilton and May 1977, Olivieri et al. 1995, Olivieri and Gouyon 1997, Ronce et al. 2000, Delgado et al. 2011), only 


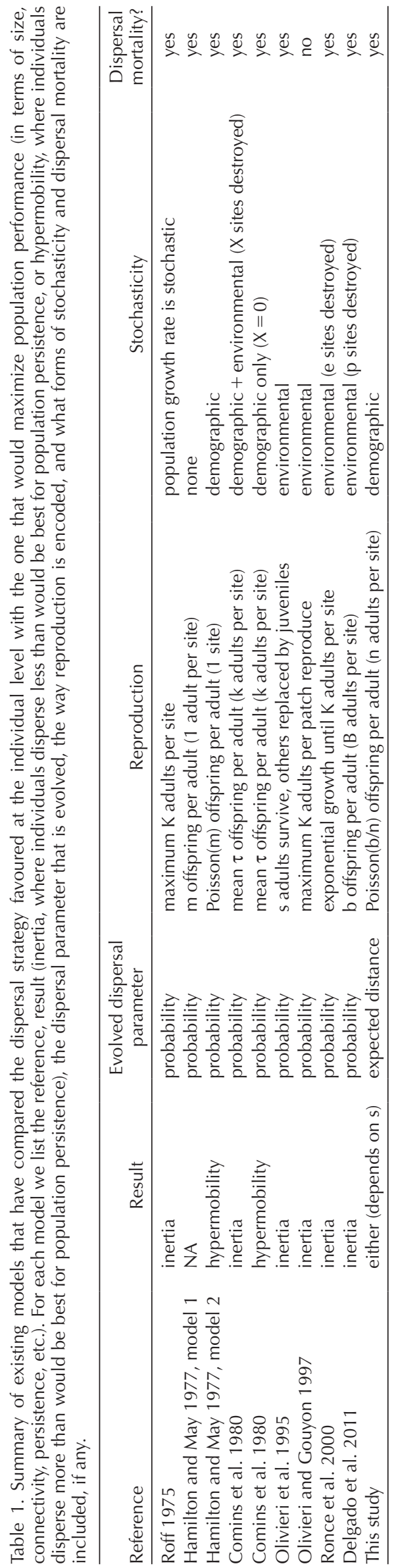

one documents both (Comins et al. 1980), and the models differ in a number of key assumptions (Table 1) that makes comparing directly across them tricky. Nevertheless Olivieri and Gouyon (1997) have suggested that hypermobility should be expected when local extinctions are due to environmental stochasticity, and inertia when local extinctions are due to demographic stochasticity.

As a consequence, we lack a theoretical understanding of when to expect evolution at the individual level to also maximize population persistence, when not, and, in the latter case, when to expect hypermobility or inertia. This is a considerable shortcoming, given the numerous conservation problems requiring sufficient connectivity for both gene flow and metapopulation persistence (Hanski and Ovaskainen 2000, Ronce and Kirkpatrick 2001, Alleaume-Benharira et al. 2006). Consider the Seychelles warbler Acrocephalus sechellensis, a species that, in 1988, only occurred on a single island in the Seychelles (having become extinct on the others). Although other islands within the Seychelles are suitable for warblers (translocated individuals developed into thriving populations; Komdeur 1997), and warblers still have the morphological capacity for inter-island dispersal (Komdeur et al. 2004), naturally occurring inter-island dispersal is extremely rare $(0.1 \%)$ in the species (Komdeur et al. 2004). It appears plausible that the isolated nature of an island archipelago like the Seychelles has selected against individuals that are too prone to disperse. As a result, populations are less connected than they could be, given the flight capacity of the species. The capacity for dispersal can also become greatly reduced over evolutionary time in conditions of isolation (e.g. loss of flight in birds, Fulton et al. 2012).

What factors might determine the risk of connectivity being reduced by evolution? Population persistence is known to be difficult in fragmented habitats (Hanski 1999); it is likewise difficult if the total habitable area is small (Soulé 1987, Hanski 1999). Intuitively, these might also be the very situations where evolution exacerbates problems of connectivity. In worlds of small size, most habitat is near an edge, creating an expectation that selection works against individuals that attempt to move and cannot find a new habitat beyond the edge. If this combines with a fragmented nature of the existing habitat, sufficient dispersal - despite the risk of some individuals moving beyond the edge of the inhabitable world - is required for long-term population persistence.

Both empirical (Bonte et al. 2003, Schtickzelle et al. 2006, Cheptou et al. 2008) and theoretical (Travis and Dytham 1999, Heino and Hanski 2001, Baskett et al. 2007, Bonte et al. 2010) studies have shown that fragmentation can favour reduced dispersal (but with the caveat that fragmentation that increases temporal variability in survival can also lead to increased dispersal, Johnson and Gaines 1990, Heino and Hanski 2001). Intriguingly, there appears to be a lack of theoretical work that considers edges related to small total habitat area. The real world is full of edges (Saunders et al. 1991, Fahrig 2003), and these are known to potentially influence dispersal (e.g. flightlessness in birds is more common on islands; Roff 1994, see Fulton et al. 2012 for a case study), yet models of dispersal are often explicitly constructed to avoid edge effects (but see Gros et al. 2006). Therefore, studies to date have not explicitly asked whether a small total size of the inhabitable area (which implies risky 
dispersal as most long-distance dispersal events prove futile) resembles fragmentation in that both might provoke inertia. If so, this could indicate that conservation efforts might even include assisted movement of individuals (Baguette et al. 2013).

Any assessment of evolution's impact on connectivity must also consider individual-level variability in dispersal (Baguette et al. 2013). Adaptation cannot occur without variation that selection can act on, and further, the spatial spread of a population will greatly depend on such variation. Past studies have suggested that heterogeneity across individuals in dispersal behaviour can lead to leptokurtic dispersal distances (Skalski and Gilliam 2000) with increased long-distance dispersal events. Given the importance of long-distance dispersal for population persistence and spread (Kot et al. 1996, Baguette 2003, Kremer et al. 2012), it seems intuitive that heterogeneity in dispersal strategies should generally increase connectivity, especially as genetic variation in dispersal leads to the potential for spatial evolution, e.g. a spatial sorting of fast-moving individuals near an invasion front (Phillips et al. 2010). Yet despite evidence that individual variation in dispersal is common (Clobert et al. 2009), theoretical studies tend to focus on mean dispersal behaviour for simplicity.

Here we derive predictions on how evolution changes the connectivity of a population by considering individual dispersal (both average and variation in) and tracking its population-level outcomes. Following Delgado et al. (2011), we are particularly interested in whether individuals disperse less (inertia) or more (hypermobility) than what would be best for population performance, measured as either total population size or fraction of the habitat occupied. We consider two levels of habitat boundaries, so that the world consists not only of discrete patches but is also limited in its total size. This creates local edges between patches as well as a global edge beyond which no suitable habitat can be found (Fig. 1).

Our results show, in line with previous work (Delgado et al. 2011), that the evolved dispersal strategy is rarely the one that maximizes population performance. One novel finding is a clear relationship between inertia and the type of a 'world' that an organism lives in: populations that are most at risk (small worlds, small patch carrying capacities, and high dispersal mortality) tend to display inertia whereas large worlds, large patch carrying capacities, and low dispersal mortality shift the outcome towards hypermobility. We also show that although heterogeneity in individual dispersal strategy can increase population connectivity, this is not guaranteed to be true. Increased heterogeneity (with no change in mean dispersal) can also imply that a larger proportion of individuals never leave their natal patch while some disperse very far, and as a whole this can impact population connectivity in a negative way.

\section{The model}

We assume a population of asexual individuals that inhabit a circular world. The world is made up of a collection of patches whose centres are randomly located within a continuous two-dimensional space, in our case with an average density of one patch per unit area (Fig. 1). There is a global

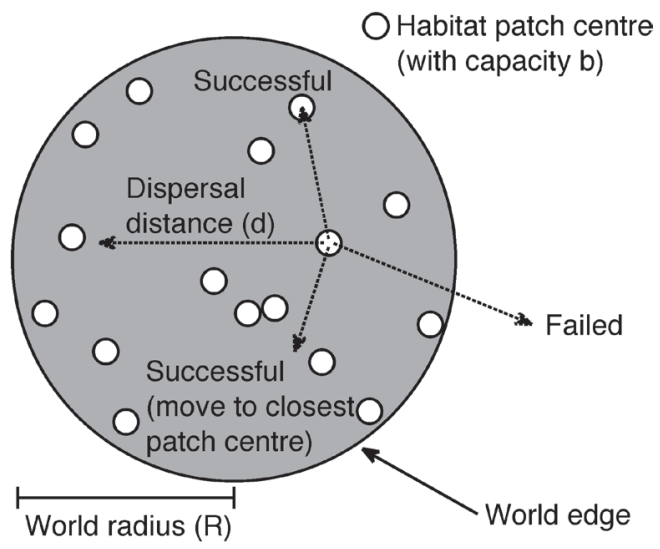

Figure 1. Model schematic. Individuals inhabit a circular world of radius $\mathrm{R}$ (large circle) made up of habitat patches, each with $\mathrm{b}$ breeding resources, whose centres (smaller circles) are randomly distributed with density 1 . Individuals disperse a distance $d$ that is drawn from a dispersal kernel with mean $\hat{d}$, and those individuals that disperse beyond the global edge of the world die.

edge with radius $\mathrm{R}$ ('world size'), beyond which no suitable patches occur. The shape of each patch is not explicitly modelled, instead each patch is simply assumed to have b breeding resources, such that there is an average of $b$ offspring produced per patch (see reproduction details below). The total carrying capacity of the world $(\mathrm{K})$ is then approximately $\pi b R^{2}$. The area outside the world is uninhabitable and any individual that disperses there dies.

Each simulation is initialized with a number of individuals $\left(\mathrm{N}_{0}=0.5 \mathrm{~K}\right)$ randomly distributed among the patches. Generations are discrete and non-overlapping - in each generation, all newborn individuals disperse, reproduce and then die. If an individual dispersed successfully, it produces a number of offspring given by Poisson $\left(\mathrm{b} / \mathrm{n}_{\mathrm{it}}\right)$, indicating that the breeding resources $b$ of the local patch are shared among the $n_{i t}$ individuals that are currently inhabiting patch $i$ at generation $\mathrm{t}$.

Each individual is characterized by a non-negative dispersal parameter $\hat{\mathrm{d}}$, which defines its dispersal strategy. Individuals inherit their parent's value of $\hat{d}$ plus a small mutation (a Gaussian random number with mean 0 and standard deviation 0.05 ). To keep $\hat{\mathrm{d}}$ non-negative (a requirement of the dispersal kernel function), any negative values that are generated through mutation are set to be exactly zero instead. Each newborn disperses in a random direction with a distance $d$ generated from a dispersal kernel with mean $\hat{d}$. We considered two types of dispersal kernel: an exponential kernel where the probability of traveling a distance $\mathrm{x}$ is given by

$\mathrm{p}(\mathrm{x})=\left(\frac{1}{\hat{\mathrm{d}}}\right) e^{-\frac{\mathrm{x}}{\hat{\mathrm{d}}}}$

and a student's t one-parameter kernel where the probability of traveling a distance $\mathrm{x}$ is given by

$\mathrm{p}(\mathrm{x})=\frac{2 \mathrm{x}}{\mathrm{u}\left(1+\frac{\mathrm{x}^{2}}{\mathrm{u}}\right)^{2}}$ 
where

$\mathrm{u}=\sqrt{\frac{\hat{\mathrm{d}}}{1.571}}$

(Venable et al. 2008). An exponential kernel has the advantages of being simple, is one of the most commonly used kernels since it is derived from classic theory (Nathan et al. 2012) and is often a good match to empirical data (Venable et al. 2008, Hovestadt et al. 2011). However, kernels with fatter tails than the exponential often provide a better fit for long-distance data (Nathan et al. 2012), so we also used a student's t kernel, which has also been found to be a good fit to empirical data (Clark et al. 1999, Venable et al. 2008).

We assume the probability of surviving dispersal decreases with distance travelled, according to an exponential distribution with mean $s$. The parameter $s$ then describes the expected 'safe' distance that an individual can disperse and still survive.

Dispersal then has three possible outcomes. First, dispersal can fail because an individual died from dispersal mortality. Second, dispersal can lead to an individual landing outside the global edge of the world, and these individuals too are removed from the population. This leaves individuals that survive dispersal and land within the world as defined by the global edge. For each of these individuals, we record its location as that of the closest patch centre (this makes a set of individuals, all sharing the same centre, who later compete with each other). Note that an individual whose dispersal distance is small is likely to end up in the same patch where it started, thus we give individuals the option of not dispersing at all.

Individual dispersal can have repercussions at the population level in terms of size, connectivity, and persistence. While connectivity is broadly defined as the "degree to which landscape facilitates or impedes movement among resource patches" (Taylor et al. 1993, Baguette et al. 2013), it must be emphasized that it is not a property of the landscape alone, but an emergent property of the landscape structure and the dispersal traits of a given species (Baguette et al. 2013). Despite the intuitive importance of this concept, there is a surprising lack of consensus regarding metrics of connectivity within the literature (Calabrese and Fagan 2004). Therefore, we calculated two simple metrics that reflect how well a population is able to remain connected and persist: the total population size and patch occupancy (fraction of patches occupied), both measured at eco-evolutionary equilibrium. Note that while these appear sensible in the current context where dispersal is the only evolving trait, we can imagine alternative contexts where we would not recommend such simple metrics. If, for example, fecundity were under selection, positive (negative) changes in patch occupancy would not necessarily be interpretable as an increase (decrease) in connectivity.

At the start of each simulation, we first let the population spread and inhabit the world, with all individuals using the same specified value of the dispersal parameter $\hat{d}$. Note that this implies the population is homogeneous with respect to the dispersal kernel, but individuals still differ in the realized dispersal distance $\mathrm{d}$; we refer to this as the 'homogeneous' stage of simulations throughout the paper. This stage ran for 1000 generations, during which no evolution was allowed to occur. We recorded the value of the two population-level performance metrics at the end of the homogeneous stage. Thereafter, we allowed simulations to proceed for 4000 more generations with mutation and evolution (enough time to ensure that a stable distribution of genotypes has been reached), yielding an evolved value of $\hat{d}$. At the end of this 'evolutionary' stage, we recorded the average $\hat{d}$ value across the population, as well as the values of the two metrics described above. This approach allowed us to look at the effect that the evolved dispersal strategy (value of $\hat{\mathrm{d}}$ ) had at the population level, based on the two metrics.

Finally, we ran a set of simulations to separate out the role of variation in dispersal strategies across individuals, independent of evolutionary pressures or spatial population structure. In these simulations, individual $\hat{\mathrm{d}}$ values in the population were drawn from a Gaussian distribution with the same mean, and different standard deviation values across runs. Each newborn individual drew its $\hat{d}$ value from the population Gaussian distribution instead of inheriting $\hat{d}$ from its parent. This approach allowed us to ensure that the distribution of $\hat{d}$ in the population remained constant throughout the simulation and did not vary spatially. Each of these simulations was run for 1000 generations before quantifying the two population-level metrics. (Full model code is available from Dryad, Shaw et al. 2014.)

\section{Results}

\section{Evolved dispersal distance}

The evolved dispersal strategy $(\hat{\mathrm{d}})$, hereafter simply referred to as 'dispersal distance' although it technically describes the expected value of the dispersal distance distribution) was highly dependent on model parameters. As the size of the world (R) decreased, the evolved dispersal distance decreased as well (Fig. 2), indicating an evolutionary response to a higher risk of moving beyond the edge of the world during dispersal. The evolved dispersal distance was highest when the average number of offspring produced per patch (b) remained small (Fig. 2). This reflects the effects of competitive kin interactions: a small b (equivalent to poorer patch quality) leads to fewer breeding individuals per patch, and offspring staying in their natal patch are then more likely to be competing with their siblings (as opposed to offspring of unrelated breeders). The evolved dispersal distances were generally lower under the exponential kernel than under the student's t kernel (Fig. 2).

The dependence of dispersal on $\mathrm{b}$ and $\mathrm{R}$ means that the evolved dispersal distance depends on the spatial distribution of the breeding resources. For example, a world with three patches each producing 10 offspring selects for very different dispersal than a world with 10 patches that each produce three offspring, despite the fact that these worlds have the same overall carrying capacity. Overall, the evolved dispersal distances were more sensitive 

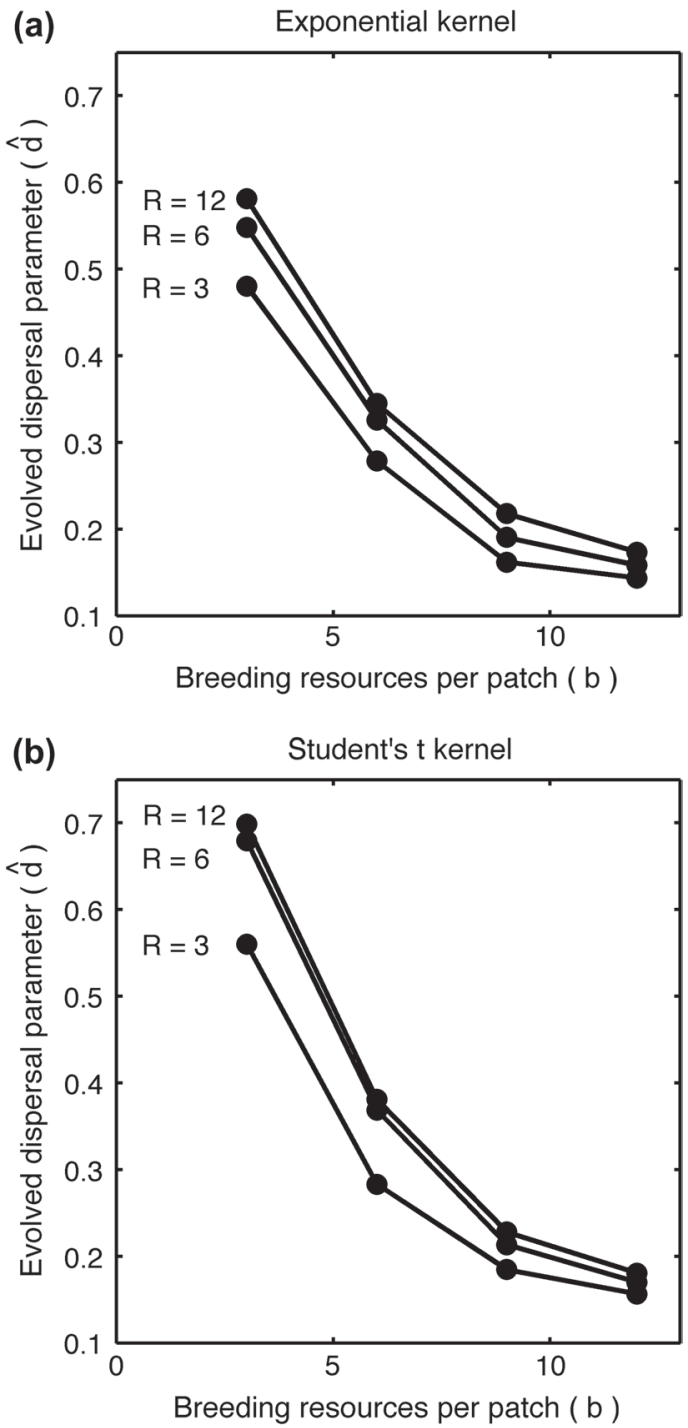

Figure 2 . The evolved dispersal distance parameter $(\hat{\mathrm{d}})$ was highly dependent on the structure of the world: it decreased as the number of breeding resources per patch (b) increased and increased as the total world size (R) increased. This was true for both simulations with the (a) exponential and (b) student's $t$ dispersal kernel. Overall, evolved dispersal distance was more sensitive to change in $b$ than changes in $R$. Dots show the average value of $\hat{d}$ across individuals in the simulated population after 5000 generations. Parameter values: $s=3$ (high safe dispersal distance, low dispersal mortality).

to the value of $\mathrm{b}$ than $\mathrm{R}$ (Fig. 2). As the 'safe' dispersal distance $(\mathrm{s})$ increased, the evolved dispersal distance also increased (Fig. 3, filled dots). See Supplementary material Appendix 1 Fig. A1 and A2 for results across all of parameter space.

\section{Hypermobility or inertia?}

For each simulation, we determined the dispersal distance that would maximize either of two population-level performance metrics (population size and patch occupancy). All else being equal, higher values of either metric can be assumed to indicate better connectivity and population persistence (Soulé 1987, Hanski 1999). Generally the dispersal distance that maximized population persistence, as measured by these proxies, showed the same trends with respect to model parameters $R, b$, and $s$ as the evolved dispersal distance (Supplementary material Appendix 1 Fig. A1-2).

Generally, the evolved dispersal strategy was not the strategy that would maximize either of these metrics, although coincidental maximization did sometimes occur. When dispersal had no mortality cost, the only reason for dispersal failure is dispersal beyond the global edge, because all dispersing individuals that land inside the world are assumed to survive. In such a setting, we always found hypermobility, i.e. populations would reach higher sizes and better patch occupancy if individuals dispersed less (indicated by the dashed line being to the left of the solid line in Fig. 3a and c, using population size as a measure). Introducing and increasing dispersal mortality cost (implemented by decreasing the average safe dispersal distance $s$ ) led to a shift from hypermobility (Fig. 3a, c) to inertia (Fig. 3b, d; now the dashed line is to the right of the solid line). See Supplementary material Appendix 1 Fig. A1 and A2 for results across all of parameter space.

In other words, when dispersal costs are high, population performance, measured as total size, would increase if individuals dispersed more. We can consider the difference between the maximum population size and the evolved population size a measure of the population 'cost' of evolution. This was highest in small populations (low b, low R; not shown).

The value of $s$ where the transition between hypermobility and inertia occurred is also the location where the evolved dispersal strategy coincides with that maximizing population performance. The location of this point of 'evolved optimality' depended on the other model parameters. Whether we used population size or patch occupancy as a metric, it occurred at higher dispersal mortality cost (lower s) as either b or R increased, implying that smaller populations in more restricted habitats are more likely to suffer from inertia. The location of this point was again more sensitive to $b$ than $R$. It occurred in different locations for each of the two population-level metrics we considered: hypermobility as defined based on patch occupancy occurred across a smaller region of parameter space than hypermobility as defined based on total population size (not shown). In other words, connectivity problems of the kind where some patches are not found at all are more likely to result from inertia than are population performance problems that are measurable as total population size.

\section{The population cost of evolution: there's more to it than moving along a dispersal-performance curve}

Our results indicate that evolution can fail to find the dispersal distance that we predict to be associated with maximal population performance, whether it is measured as patch occupancy (fraction of the habitat occupied) or population size. However, the mean distance is not the only factor predicting population performance: variability also matters. This is why an evolved distribution of dispersal distances (which includes variability) does not necessarily 
Exponential kernel
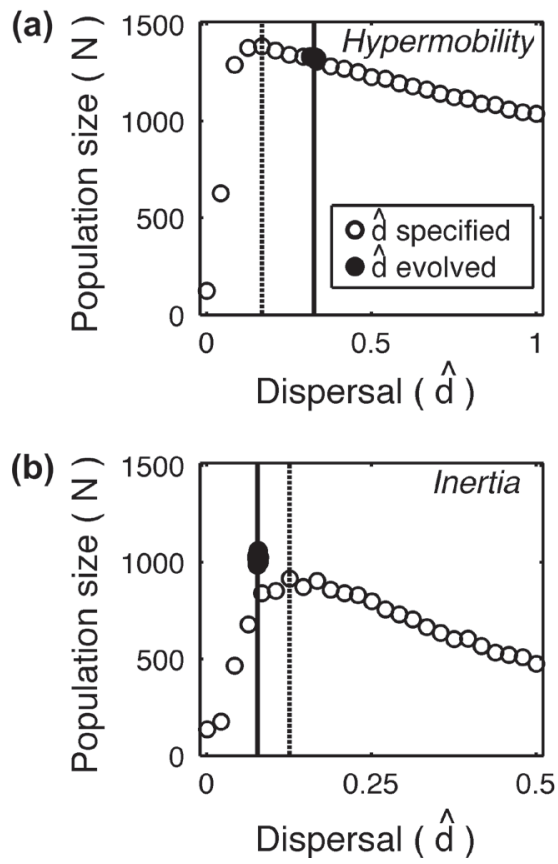
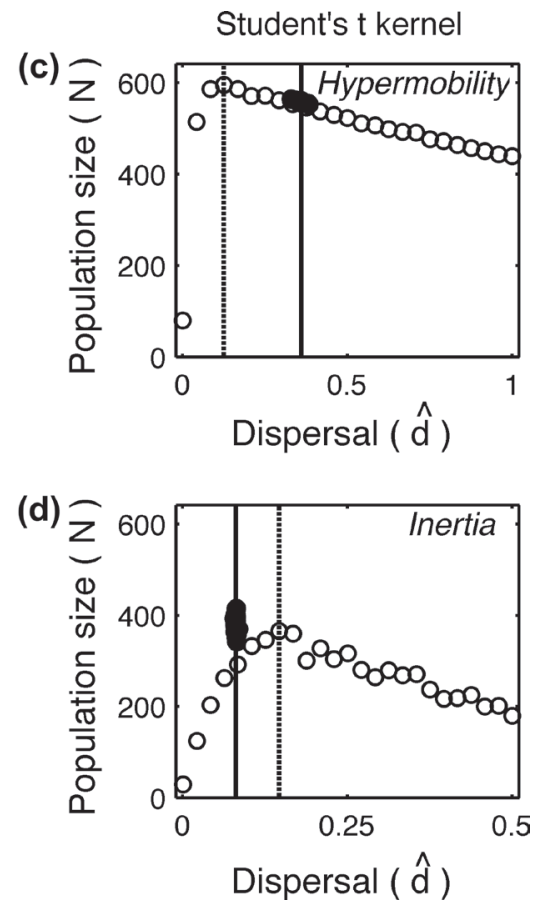

Figure 3. Hypermobility and inertia. The curve consisting of open dots shows the population size (N, one of the population-level performance metrics) after 1000 generations of population dynamics with all individuals using the same dispersal kernel with mean distance $\hat{d}$, as given on the $\mathrm{x}$-axis. Filled dots show the mean of evolved $\hat{d}$ together with the associated value of $\mathrm{N}$, for 25 initial $\hat{d}$ values. The $\hat{d}$ that maximizes $\mathrm{N}$ is indicated with a vertical dashed line, and the average $\hat{\mathrm{d}}$ across all evolved simulations is indicated with a vertical solid line. Parameter values: breeding resources $b=6 ;(a, b)$ exponential dispersal kernel with world size $R=9$ and (c, d) student's $t$ dispersal kernel with $\mathrm{R}=6$; and the distance that an individual is likely to survived is (a, c) $s=3$ (low dispersal mortality, indicating it is easy to survive the average interpatch distance of 1$)$ and (b, d) $s=0.4$ (high dispersal mortality).

produce the same population performance as that predicted by the same mean dispersal but no variability (e.g. Fig. 3b; note that the performance metric curves are from the 'homogeneous' stage of simulations where all individuals have the same expected, though not necessarily realized, dispersal distance). Heterogeneity is due primarily to the introduction of variation through mutation. Although dispersal parameter values varied spatially in our simulations, the patterns changed over the course of a simulation and hence did not seem to be due to local adaptation (Supplementary material Appendix 1 Fig. A3). There was no trend in average dispersal values as a function of distance from the edge of the world.

\section{Effect of heterogeneity in the dispersal parameter}

Since the above result highlights a potentially complicated relationship between variability in dispersal and population persistence or connectivity (Clobert et al. 2009), we further explored the discrepancy between the evolved population performance metrics and those expected based on homogeneous $\hat{d}$ (i.e. no individual variation in strategy). Different starting values of $\hat{d}$ converged to nearidentical evolved dispersal parameter values and the corresponding two population performance metrics (the filled dots, denoting evolved values, are clustered in Fig. 3 and the left panels of Fig. 4). However, the values of these metrics often differed markedly from those derived from homogeneous simulations (where all individuals had the same value of $\hat{d}$ ). Heterogeneity in individual dispersal did not always follow the pattern of Fig. $3 b$ where it increased the population performance: its effect was sometimes negative. In regions of parameter space where the dispersal distance evolved to be high (about $\hat{\mathrm{d}}>0.25$ for the exponential kernel and $\hat{\mathrm{d}}>0.35$ for the student's $t$ kernel), patch occupancy evolved to be much lower than in corresponding homogeneous simulations (filled dots fall below open dots curve in e.g. Fig. 4a). Conversely, evolution improved patch occupancy over that found in corresponding homogeneous simulations in those regions of parameter space where dispersal distance evolved to be low. The effect on population performance was present even at much lower mutation levels than those considered in our main simulations, although higher mutation levels resulted in a larger effect on the population performance metrics (Supplementary material Appendix 1 Fig. A4).

To further unpack the role of heterogeneity, we ran an additional set of simulations where dispersal parameter values did not evolve. They were instead drawn for each individual from a normal distribution using the mean of the evolved simulations in the left panels of Fig. 4, but with different fixed standard deviation values (Fig. 4, right panels). Increased individual heterogeneity in this setup can decrease patch occupancy (Fig. 4c) but also increase it (at least in a comparison against no or very little heterogeneity, Fig. $4 \mathrm{~d}, \mathrm{~h}$ ). Note that as the standard deviation increases, there is an increase in both the shortest and longest dispersal distances. The lengthening of the dispersal 
Exponential kernel
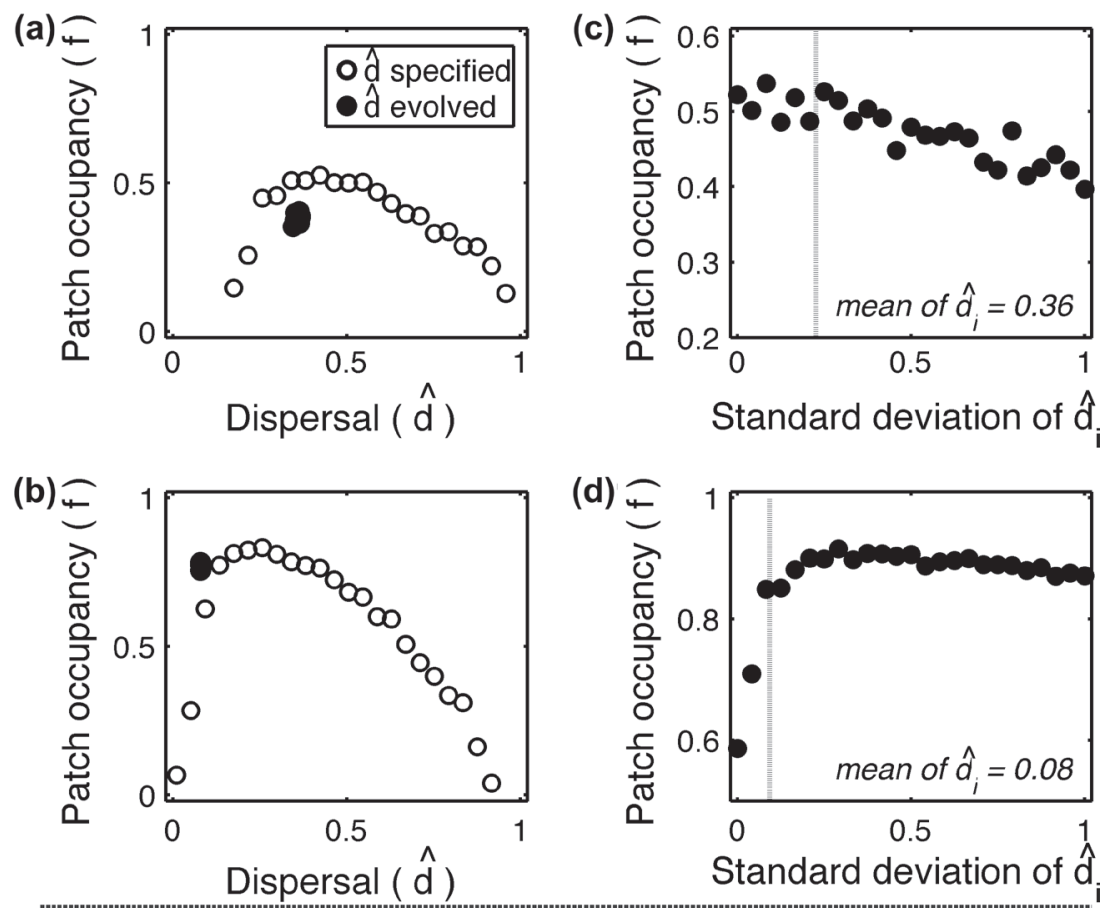

Student's t kernel
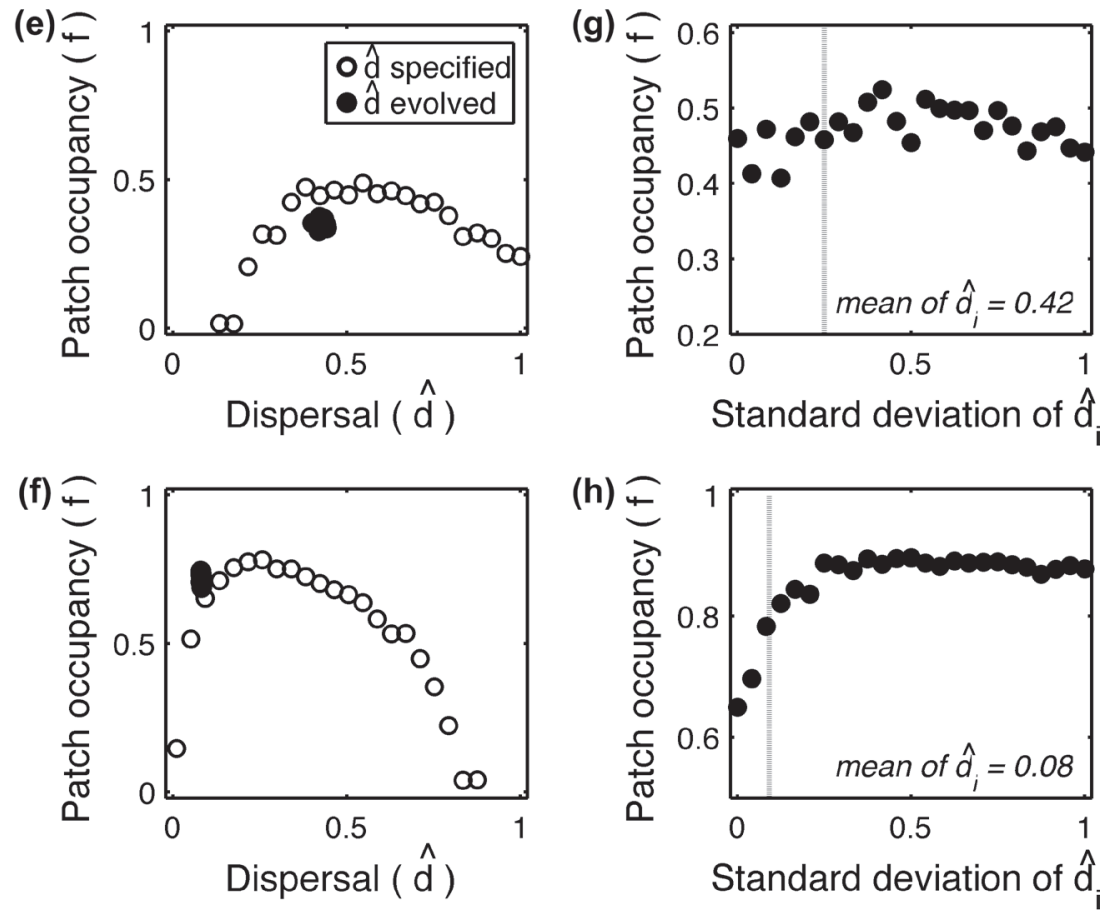

Figure 4. Positive and negative effects of heterogeneity under the (a-d) exponential and (e-h) student's t dispersal kernel. The patch occupancy ( $\mathrm{f}$, one of the population-level performance metrics) from simulations where (left panels, open dots) all individuals use the same dispersal kernel with mean distance $\hat{\mathrm{d}}$ (x-axis), (left panels, filled dots) the mean dispersal parameter d evolves (see Fig. 3 for more details), and (right panels) each individual's $\hat{\mathrm{d}}$-value is drawn from a population Gaussian distribution with fixed mean. Parameter values: world size $\mathrm{R}=12 ;(\mathrm{a}, \mathrm{c}, \mathrm{e}, \mathrm{g})$ breeding resources $\mathrm{b}=3$ and safe dispersal distance $s=1$, and $(\mathrm{b}, \mathrm{d}, \mathrm{f}, \mathrm{h}) \mathrm{b}=6$ and $s=0.4$. The mean values of $\hat{\mathrm{d}}$ in the right panels are set as the average evolved values from each of the left panels: $0.36,0.08,0.42$ and 0.08 , respectively. The lines in the right panels show the actual standard deviation from the filled dots in each of the left panels. In (a, e), the patch occupancy, $\mathrm{f}$, is much lower if the dispersal parameter evolves and therefore is heterogeneous in the population than if dispersal is specified to be homogeneous in the population. In $(b, f)$, the opposite is true. These differences reflect the effects of heterogeneity, which has opposite effects in different regions of parameter space (see also Fig. 5). 
distance has a disproportionately important effect on the dynamics, since short dispersal distances are bounded by 0 , while ever longer dispersal attempts lead to ever higher mortality rates (from both the probability of dying en route as well as the probability of dispersing beyond the edge of the world). This effect likely causes the decreased population performance for high standard deviation values in Fig. 4d.

The fact that heterogeneity can either act to increase or decrease patch occupancy suggests that heterogeneity in dispersal (introduced in our evolutionary simulations via mutation) changes the likelihood of individuals moving between patches. These inter-patch movements are most likely for intermediate dispersal events: distances that are long enough to bring an individual to a new patch, but not so long that population connectivity deteriorates because the individual dies or disperses beyond the edges of the habitable world (the two costs mentioned above).

This implies that a too strong focus on the tail of the dispersal kernel (which suggests that longest dispersal events are disproportionally important for population performance) might be misleading: a heterogeneous strategy might have relatively few individuals performing intermediate dispersal (i.e. leaving the patch but not necessarily going far), and such individuals can be important. We can explore the probability of a new patch being found analytically for a simpler, more generalized model.

Consider a population of $\mathrm{N}$ individuals with an exponential dispersal distance, such that the ith individual disperses a distance given by $d \sim \operatorname{Exp}\left(\hat{\mathrm{d}}_{\mathrm{i}}\right)$. The fraction of dispersal events that will take an individual outside of its natal patch (dispersal distance longer than a threshold distance $\mathrm{k}$ ) is given by

$f=\frac{1}{N} \sum_{i=1}^{N} e^{-\frac{k}{d_{i}}}$

Calculating this fraction numerically for a range of different lognormal distributions of $\hat{\mathrm{d}}_{\mathrm{i}}$, we find that it is highly sensitive to the mean and variance of dispersal parameter values in the population, $\hat{\mathrm{d}}_{\mathrm{i}}$, as well as the threshold distance (Fig. 5). If the average distance between patches is, for example, 10, it is of interest to examine how often dispersal is longer than $\mathrm{k}=5$ (long enough to typically bring an individual to another patch) versus less than $\mathrm{k}=5$ (keeping it in its natal patch).

When the average dispersal distance is low, heterogeneity in dispersal parameter values increases the probability of a dispersal event that is long enough to land the individual outside its natal patch (e.g. Fig. 5, top row), thus it is likely to increase population connectivity and persistence. This improvement explains why patch occupancy can evolve to be higher than what is expected based on homogeneous simulations (e.g. Fig. 4b). However, when dispersal distances are higher, heterogeneity in dispersal parameter values lowers the probability of patch switching (e.g. Fig. 5, bottom row). This reduced probability is the key factor when understanding why evolution can lead to decreased connectivity (measured as lower patch occupancy in evolutionary simulations than in homogeneous ones e.g. in Fig. 4a). Note that in both of these cases, increasing heterogeneity still increases the probability of the longest dispersal events (Fig. 5e, f, dispersing above $\mathrm{k}=10$ ).

These results seem to be robust to the choice of distributions: we obtain qualitatively the same result if we assume the distribution of parameter values in the population is Gaussian, or if individuals disperse according to a halfnormal distribution instead of an exponential distribution (not shown). Note that in our simplified description (Eq. 3) the focus is on the probability of leaving a patch, which improves with dispersal. In contrast, the prospects of colonizing a new patch can potentially decrease with increasing dispersal distance as explained above, particularly strongly so if the total area of inhabitable habitat is small. Taking all these factors together, we can explain why heterogeneity in dispersal behaviour can, very generally, either increase or decrease connectivity (and in turn,
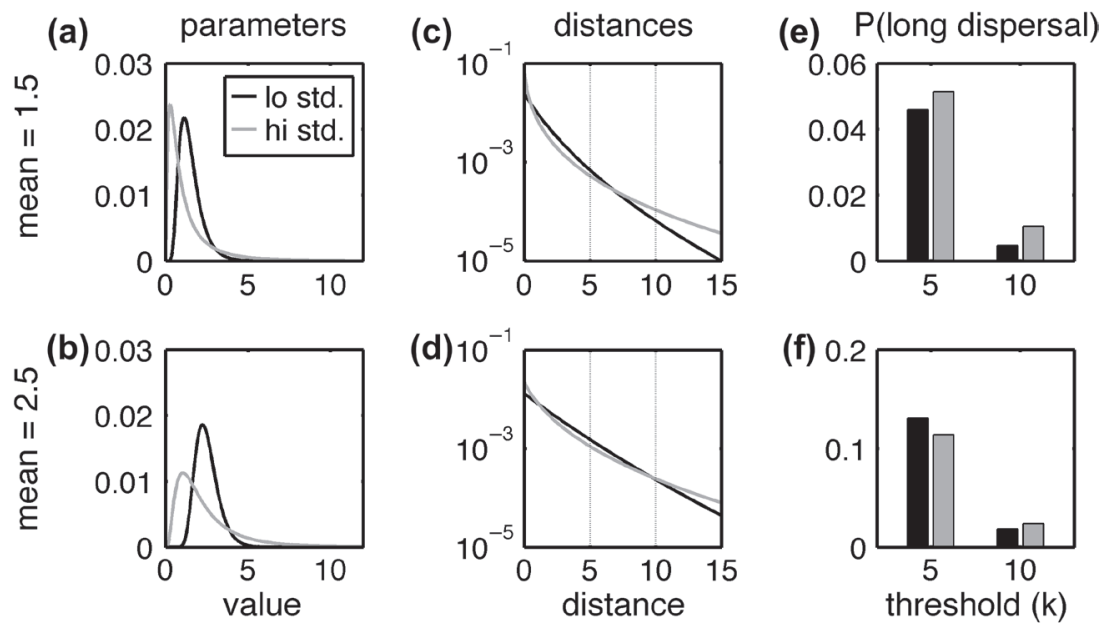

Figure 5. Effect of dispersal mean and variance on the fraction of dispersal events that are beyond a threshold distance. (a-b) The lognormal distribution of dispersal parameter values in the population for low (black, 0.5) and high (grey, 5) standard deviations, and for low (a, 1.5) and high (b, 2.5) average values. (c-d) The distribution of dispersal distances for each of these four cases. (e-f) The fraction of dispersal events beyond a threshold distance, $k$, for each of the four cases. Note that while increasing the variance always increases the probability of long-distance dispersal events $(\mathrm{k}=10)$, it can either increase or decrease the probability of patch-leaving dispersal events $(\mathrm{k}=5)$. 
persistence), and why this is unlikely to be an idiosyncratic outcome of our particular model assumptions about habitat structure, mortality, or dispersal kernel.

\section{Discussion}

Our model is in line with earlier work confirming that dispersal, while being the 'glue' that keeps populations connected, does not necessarily evolve to have properties that maximize population connectivity and persistence. Here we present two sets of novel findings. First, we show that the structure of the 'world' individuals inhabit is an important determinant of whether individuals are selected to avoid dispersal to the extent that population persistence becomes compromised. This is much more likely when the habitat is both small and fragmented than in larger 'worlds', which is worrisome as these are populations experiencing the more severe persistence problems in the first place. Second, we demonstrate that there is an underappreciated force at play: dispersal trait evolution cannot occur without individual differences in dispersal strategy, but this heterogeneity itself changes the population persistence and connectivity either positively or negatively. Below we discuss these effects in more detail.

\section{World structure}

Before quantifying the impact of individual dispersal behaviour at the population level, we first confirmed that the trends of dispersal evolution in our model fit with past dispersal models. This appears particularly important since we considered the evolution of dispersal distance (with individuals not dispersing beyond their natal patch if this remained small), while much of dispersal literature has worked on dispersal rates (probabilities of leaving).

Our findings are in line with those of Gros et al. (2006) that dispersal distance increases as world size increases, since this diminishes the risk of dispersing beyond an edge. This indicates that constraining populations to smaller areas than before (e.g. due to anthropogenic habitat modifications) could have detrimental implications for dispersal and gene flow, even if the habitat mosaic inside the core area remains entirely intact. Likewise, we predict that populations that have inhabited isolated areas for long periods can be particularly vulnerable to fragmentation and/or loss of individuals from some sites. The tendency for Seychelles warblers to avoid dispersing to neighbouring islands is a good potential example: habitat restoration is in such cases not sufficient; dispersal also had to be assisted to reach current levels of patch occupancy and population size.

Our results also match the findings of Comins et al. (1980) and Rousset and Gandon (2002) that smaller local carrying capacities favour dispersal, and the findings of Poethke et al. (2003) and Delgado et al. (2011) that higher dispersal mortality usually selects against dispersal. While we initially expected that the evolved dispersal distance would be highly sensitive to the probability of dispersing beyond the global edge of the world, instead it was more sensitive to the number of offspring produced per patch (b). Low b implies that philopatry would often lead to competing with related individuals, which can strongly promote dispersal. Although the result of kin selection favouring dispersal is by no means new (Hamilton and May 1977, Frank 1986, Gandon and Rousset 1999, Ronce 2007, Kubisch et al. 2013), this result highlights its relative importance in comparison to other model parameters. Delgado et al. (2011) and Comins et al. (1980) both found that dispersal probability increased slightly for very high mortality. We did not find this effect in our model, although such an 'evolutionary rescue' effect is often sensitive to model assumptions and parameters (Kokko 2007); in earlier work it has only occurred in model versions that included random patch destruction (Comins et al. 1980).

\section{Hypermobility or inertia: risky dispersal leads to the latter}

Based on earlier work (Delgado et al. 2011), our finding that evolved dispersal strategies rarely maximize populationlevel performance metrics was not surprising. By contrast, our second finding, that there is a transition from hypermobility (population persistence would increase if individuals dispersed less) to inertia (population persistence would increase if individuals dispersed more) as dispersal mortality increases, is an unexpected result that has not been mentioned in any of the existing dispersal models that included dispersal mortality (Table 1). Note, however, that Comins et al. (1980) documented a switch from hypermobility to inertia as patch destruction was added to their dispersal model.

Olivieri and Gouyon (1997) suggested that hypermobility arises when local extinctions are due to environmental stochasticity, and that inertia arises when local extinctions are due to demographic stochasticity. Our results demonstrate that it is, in fact, possible to get both hypermobility and inertia in the absence of any environmental stochasticity. Our assumptions differ from earlier modelling in that we assume a stable world (all patches are always suitable), but we include two different risks of dispersal: a mortality cost of moving as well as a risk of dispersing beyond the global edge. This means that we can set the mortality cost to be very low or even zero without simultaneously removing all cost there is to dispersing, and these conditions allow hypermobility to occur in a stable world.

Our results fit the intuition of Delgado et al. (2011): we find that inertia is more common in population that are most at risk - those that inhabit small worlds, have small patch carrying capacities, and high dispersal mortality. See Supplementary material Appendix 2 for detailed intuition for why this is the case. Our results also provide some important qualifications for the prediction made by Delgado et al. (2011) that inertia should be more common than hypermobility. Within the parameter region we studied, we found hypermobility more often than inertia. However, any evaluation of the likelihood of a certain outcome in nature should also take into account the likely prevalence of the parameter values assumed. In reality, dispersal is costly (Bonte et al. 2012), and since inertia occurs in our models when these costs are significant, it may be that most real-world examples fall into this region of parameter space. Somewhat worryingly for a conservation 
context, low birth rate (low b) increases the conservation concern for a species, and it is also simultaneously predicted to increase the likelihood of inertia, suggesting the need for assisted migration.

\section{Dispersal heterogeneity}

Clobert et al. (2009) recently highlighted the importance of accounting for individual differences in dispersal behaviour. It is tempting to assume that heterogeneity in dispersal strategies is always beneficial, given past studies indicating that dispersal heterogeneity can create leptokurtic dispersal kernels, which are known to lead to increased population spread and persistence (Skalski and Gilliam 2000, Kot et al. 1996). Our findings confirm that increased heterogeneity in dispersal parameter values across individuals leads to leptokurtic dispersal kernels and that this does increase dispersal above a threshold distance - but this is only uniformly true for very high threshold distances. When considering thresholds that are lower (e.g. the probability of leaving one's natal patch), the opposite can hold; leptokurtic kernels decrease the probability of dispersal above those thresholds. In other words, leptokurtosis (with its 'fat tails' and 'high peaks') can simultaneously increase very long-distance dispersal but also decreases the probability of switching to another patch at all. Because modest dispersal distances are also the safest ones (the probabilities of dying en route as well as dispersing beyond the edge of the world remain small), heterogeneity can lead to lower connectivity and poorer population-level occupancy if dispersal is too small-scale to begin with.

\section{Caveats and future directions}

Our model includes a number of simplifying assumptions that could be relaxed in future general studies on hypermobility and inertia. First, our use of one-parameter dispersal kernels inherently links dispersal distance and emigration rate. Our finding that different average dispersal distances evolved under different dispersal kernels indicates that dispersal distance is not the only aspect of dispersal under selective pressure. Indeed past theoretical studies have highlighted the importance of considering the evolution of dispersal rate and distance separately (Murrell et al. 2002, Rousset and Gandon 2002). Future studies could unpack the relative roles of dispersal rate and distance in leading to inertia and hypermobility. Second, to minimize the number of model parameters we assumed that each patch has a fixed amount of breeding resources that is split among breeding individuals, which inherently links the strength of local competition and individual reproductive rate together. Future work could usefully consider two separate parameters to separate out the effects of competition strength and colonization probability. Finally, we assume that dispersal follows a predetermined statistical distribution of distances. While kernels are useful as a basic description of dispersal, the distribution of dispersal distances is, in reality, an emergent property of the choices individual make during the three phases (immigration, transit, settlement) of dispersal (Nathan et al. 2012). Future models that explicitly consider one or more of these stages (Travis et al. 2012) could test whether inertia and hypermobility still emerge in these more complex descriptions of dispersal.

Our model was built with generality in mind, thus we did not include assumptions that would associate very closely with any particular mode of dispersal; the life history modelled was also kept minimalistic for this reason. Our assumptions could therefore be adjusted to be considerably more system-specific in future studies aimed at applying our findings to a particular species. For example, a model version for plant passive dispersal might consider each patch as the location of a single adult plant, model the mortality process as removal of an adult, and allow dispersing individuals to only establish in unoccupied patches. In contrast, a model version for bird dispersal might consider each patch to be the centre of a nesting territory, incorporate details on mating system and sex-specific decisions, and allow individuals that dispersed off the 'edge' of the habitat the option of dispersing back, if they turn around in a short enough timeframe. Finally, we chose total population size and patch occupancy (fraction of patches occupied), as metrics of population performance (connectivity and persistence). However, we stress that these will not necessarily be the best metrics in other models or empirical studies, and recommend that future studies choose metrics based on the system of interest.

To conclude, evolution can impact population connectivity and persistence in a variety of ways. In addition to there being 'too little' or 'too much' dispersal, evolved dispersal can also be too heterogeneous or too homogeneous, if in each case we rate the ideal according to the perspective that a manager of a population would take (i.e. improved population connectivity and persistence). Intriguingly, the problems appear to be worst for those populations that are already at highest risks: populations with a low birth rate and costly dispersal, inhabiting fragmented habitats of a small total geographic area, are likely to exhibit inertia, with negative consequences for gene flow and connectivity. These populations are simultaneously negatively affected by dispersal heterogeneity, adding weight to the conclusion that the mismatch between evolved and population-performance-maximizing dispersal has to be very carefully watched for in endangered populations.

Acknowledgements - This material is based upon work supported by the National Science Foundation under Grant no. OISE1159097 to AKS. HK was also supported by the Australian Research Council and HK, MJ by the Finnish Centre of Excellence in Biological Interactions. We thank M. Saastamoinen and J. Clobert for helpful comments.

\section{References}

Alleaume-Benharira, M. et al. 2006. Geographical patterns of adaptation within a species' range: interactions between drift and gene flow. - J. Evol. Biol. 19: 203-215.

Baguette, M. 2003. Long distance dispersal and landscape occupancy in a metapopulation of the cranberry fritillary butterfly. - Ecography 26: 153-160.

Baguette, M. et al. 2013. Individual dispersal, landscape connectivity and ecological networks. - Biol. Rev. 88: 310-326.

Baskett, M. L. et al. 2007. The evolution of dispersal in reserve networks. - Am. Nat. 170: 59-78. 
Bonte, D. et al. 2003. Low propensity for aerial dispersal in specialist spiders from fragmented landscapes. - Proc. R. Soc. B 270: 1601-1607.

Bonte, D. et al. 2010. Evolution of dispersal polymorphism and local adaptation of dispersal distance in spatially structured landscapes. - Oikos 119: 560-566.

Bonte, D. et al. 2012. Costs of dispersal. - Biol. Rev. 87: 290-312.

Burgess, S. C. et al. 2012. How do dispersal costs and habitat selection influence realized population connectivity? - Ecology 93: 1378-1387.

Calabrese, J. M. and Fagan, W. F. 2004. A comparison-shopper's guide to connectivity metrics. - Front. Ecol. Environ. 2: 529-536.

Cheptou, P. O. et al. 2008. Rapid evolution of seed dispersal in an urban environment in the weed Crepis sancta. - Proc. Natl Acad. Sci. USA 105: 3796-3799.

Clark, J. S. et al. 1999. Seed dispersal near and far: patterns across temperate and tropical forests. - Ecology 80: 1475-1494.

Clobert, J. et al. 2009. Informed dispersal, heterogeneity in animal dispersal syndromes and the dynamics of spatially structured populations. - Ecol. Lett. 12: 197-209.

Clobert, J. et al. 2012. Dispersal ecology and evolution. - Oxford Univ. Press.

Comins, H. N. et al. 1980. Evolutionarily stable dispersal strategies. - J. Theor. Biol. 82: 205-230.

Delgado, M. D. M. et al. 2011. Inertia: the discrepancy between individual and common good in dispersal and prospecting behaviour. - Biol. Rev. 86: 717-732.

Fahrig, L. 2003. Effects of habitat fragmentation on biodiversity. - Annu Rev. Ecol. Evol. Syst. 34: 487-515.

Frank, S. A. 1986. Dispersal polymorphisms in subdivided populations. - J. Theor. Biol. 122: 303-309.

Fulton, T. L. et al. 2012. Multiple losses of flight and recent speciation in steamer ducks. - Proc. R. Soc. B 279: 2339-2346.

Gandon, S. and Rousset, F. 1999. Evolution of stepping-stone dispersal rates. - Proc. R. Soc. B 266: 2507-2513.

Gros, A. et al. 2006. Evolution of local adaptations in dispersal strategies. - Oikos 114: 544-552.

Gyllenberg, M. et al. 2002. Evolutionary suicide and evolution of dispersal in structured metapopulations. - J. Math. Biol. 45: 79-105.

Hamilton, W. D. and May, R. M. 1977. Dispersal in stable habitats. - Nature 269: 578-581.

Hanski, I. 1999. Metapopulation ecology. - Oxford Univ. Press.

Hanski, I. and Ovaskainen, O. 2000. The metapopulation capacity of a fragmented landscape. - Nature 404: 755-758.

Heino, M. and Hanski, I. 2001. Evolution of migration rate in a spatially realistic metapopulation model. - Am. Nat. 157: 495-511.

Hovestadt, T. et al. 2011. Do all inter-patch movements represent dispersal? A mixed kernel study of butterfly mobility in fragmented landscapes. - J. Anim. Ecol. 80: 1070-1077.

Johnson, M. L. and Gaines, M. S. 1990. Evolution of dispersal: theoretical models and empirical tests using birds and mammals. - Annu. Rev. Ecol. Syst. 21: 449-480.

Kokko, H. 2007. Modelling for field biologists (and other interesting people). - Cambridge Univ. Press.

Komdeur, J. 1997. Inter-island transfers and population dynamics of Seychelles warblers. - Bird Conserv. Int. 7: 7-26.

Komdeur, J. et al. 2004. Why Seychelles warblers fail to recolonize nearby islands: unwilling or unable to fly there? - Ibis 146: 298-302.

Kot, M. et al. 1996. Dispersal data and the spread of invading organisms. - Ecology 77: 2027-2042.

Kremer, A. et al. 2012. Long-distance gene flow and adaptation of forest trees to rapid climate change. - Ecol. Lett. 15: 378-392.

Supplementary material (available as Appendix oik-01229 at

$<$ www.oikosjournal.org/readers/appendix $>$ ). Appendix 1-2.
Kubisch, A. et al. 2013. Kin competition as a major driving force for invasions. - Am. Nat. 181: 700-706.

Murrell, D. et al. 2002. The evolution of dispersal distance in spatially-structured populations. - Oikos 97: 229-236.

Nathan, R. et al. 2012. Dispersal kernels: review. - In: Clobert, J. et al. (eds), Dispersal ecology and evolution. Oxford Univ. Press, pp. 187-210.

Olivieri, I. and Gouyon, P.-H. 1997. Evolution of migration rate and other traits. - In: Hanski, I. and Gilpin, M. E. (eds), Metapopulation biology: ecology, genetics and evolution. Academic Press, pp. 293-323.

Olivieri, I. et al. 1995. Metapopulation genetics and the evolution of dispersal. - Am. Nat. 146: 202-228.

Phillips, B. L. et al. 2010. Life-history evolution in rangeshifting populations. - Ecology 91: 1617-1627.

Poethke, H. J. et al. 2003. Local extinction and the evolution of dispersal rates: causes and correlations. - Am. Nat. 161: 631-640.

Poethke, H. J. et al. 2011. A metapopulation paradox: partial improvement of habitat may reduce metapopulation persistence. - Am. Nat. 177: 792-799.

Roff, D. A. 1975. Population stability and the evolution of dispersal in a heterogeneous environssment. - Oecologia 19: 217-237.

Roff, D. A. 1994. The evolution of flightlessness: is history important? - Evol. Ecol. 8: 639-657.

Ronce, O. 2007. How does it feel to be like a rolling stone? Ten questions about dispersal evolution. - Annu Rev. Ecol. Evol. Syst. 38: 231-253.

Ronce, O. and Kirkpatrick, M. 2001. When sources become sinks: migrational meltdown in heterogeneous habitats. - Evolution 55: 1520-1531.

Ronce, O. et al. 2000. Evolutionarily stable dispersal rates do not always increase with local extinction rates. - Am. Nat. 155: 485-496.

Rousset, F. and Gandon, S. 2002. Evolution of the distribution of dispersal distance under distance-dependent cost of dispersal. - J. Evol. Biol. 15: 515-523.

Saunders, D. A. et al. 1991. Biological consequences of ecosystem fragmentation: a review. - Conserv. Biol. 5: 18-32.

Savill, N. J. and Hogeweg, P. 1998. Spatially induced speciation prevents extinction: the evolution of dispersal distance in oscillatory predator-prey models. - Proc. R. Soc. B 265: 25-32.

Schtickzelle, N. et al. 2006. Dispersal depression with habitat fragmentation in the bog fritillary butterfly. - Ecology 87: 1057-1065.

Shaw, A. K. et al. 2014. Data from: Population-level consequences of risky dispersal. Dryad Digital Repository. doi: 105061/ dryad.j7581

Skalski, G. T. and Gilliam, J. F. 2000. Modeling diffusive spread in a heterogeneous population: a movement study with stream fish. - Ecology 81: 1685-1700.

Soulé, M. E. 1987. Introduction. - In: Soulé, M. E. (ed.), Viable populations for conservation. Cambridge Univ. Press, pp. 1-10.

Starrfelt, J. and Kokko, H. 2012. The theory of dispersal under multiple influences. - In: Clobert, J. et al. (eds), Dispersal ecology and evolution. Oxford Univ. Press, pp. 19-28.

Taylor, P. D. et al. 1993. Connectivity is a vital element of landscape structure. - Oikos 68: 571-573.

Travis, J. M. J. and Dytham, C. 1999. Habitat persistence, habitat availability and the evolution of dispersal. - Proc. R. Soc. B 266: 723-728.

Travis, J. M. J. et al. 2012. Modelling dispersal: an eco-evolutionary framework incorporating emigration, movement, settlement behaviour and the multiple costs involved. - Methods Ecol. Evol. 3: 628-641.

Venable, D. L. et al. 2008. Seed dispersal of desert annuals. - Ecology 89: 2218-2227. 